\title{
GENERAL ORDER MULTIVARIATE PADÉ APPROXIMANTS FOR PSEUDO-MULTIVARIATE FUNCTIONS. II
}

\author{
PING ZHOU, ANNIE CUYT, AND JIEQING TAN
}

AbSTRACT. Explicit formulas for general order multivariate Padé approximants of pseudo-multivariate functions are constructed on specific index sets. Examples include the multivariate forms of the exponential function

$$
E(\underline{x})=\sum_{j_{1}, j_{2}, \ldots, j_{m}=0}^{\infty} \frac{x_{1}^{j_{1}} x_{2}^{j_{2}} \cdots x_{m}^{j_{m}}}{\left(j_{1}+j_{2}+\cdots+j_{m}\right) !},
$$

the logarithm function

$$
L(\underline{x})=\sum_{j_{1}+j_{2}+\cdots+j_{m} \geq 1} \frac{x_{1}^{j_{1}} x_{2}^{j_{2}} \cdots x_{m}^{j_{m}}}{j_{1}+j_{2}+\cdots+j_{m}},
$$

the Lauricella function

$$
F_{D}^{(m)}\left(a, 1, \ldots, 1 ; c ; x_{1}, \ldots, x_{m}\right)=\sum_{j_{1}, j_{2}, \ldots, j_{m}=0}^{\infty} \frac{(a)_{j_{1}+\cdots+j_{m}}}{(c)_{j_{1}+\cdots+j_{m}}} x_{1}^{j_{1}} \cdots x_{m}^{j_{m}},
$$

and many more. We prove that the constructed approximants inherit the normality and consistency properties of their univariate relatives. These properties do not hold in general for multivariate Padé approximants. A truncation error upperbound is also given.

\section{INTRODUCTION}

In [6] and [1], we explicitly construct multivariate Padé approximants to socalled pseudo-multivariate functions of two variables by using their one variable projections. Our aim in this paper is to first write a pseudo-multivariate function of at least two variables as a finite sum of one variable projections by means of divided differences, and then explicitly construct multivariate Padé approximants to the given pseudo-multivariate function. We prove that the constructed approximants inherit the normality and consistency properties of their univariate relatives, which do not hold in general for multivariate Padé approximants, and present a truncation error upperbound.

Received by the editor August 10, 2007 and, in revised form, September 5, 2008.

2000 Mathematics Subject Classification. Primary 41A21.

Key words and phrases. Multivariate Padé approximants, pseudo-multivariate functions.

The first author's research is supported by NSERC of Canada.

The second author is Research Director of FWO-Vlaanderen.

The third author's research is supported by the National Natural Science Foundation of China under Grant No. 60473114. 
Definition 1.1. Let

$$
F(\underline{x}):=F\left(x_{1}, \ldots, x_{m}\right):=\sum_{j_{1}, \ldots, j_{m}=0}^{\infty} c_{j_{1} \cdots j_{m}} x_{1}^{j_{1}} \cdots x_{m}^{j_{m}}, \quad c_{j_{1} \cdots j_{m}} \in \mathbb{C}
$$

be a formal power series, and let $M, N, E$ be finite index sets in $\mathbb{N} \times \cdots \times \mathbb{N}=\mathbb{N}^{m}$. An $(M, N)$ general order multivariate Padé approximant to $F(\underline{x})$ on the set $E$ is a rational function

where the polynomials

$$
[M / N]_{E}(\underline{x}):=\frac{P(\underline{x})}{Q(\underline{x})}
$$

$$
\begin{aligned}
& P(\underline{x}):=\sum_{\left(j_{1}, \ldots, j_{m}\right) \in M} a_{j_{1} \cdots j_{m}} x_{1}^{j_{1}} \cdots x_{m}^{j_{m}}, \quad a_{j_{1} \cdots j_{m}} \in \mathbb{C}, \\
& Q(\underline{x}):=\sum_{\left(j_{1}, \ldots, j_{m}\right) \in N} b_{j_{1} \cdots j_{m}} x_{1}^{j_{1}} \cdots x_{m}^{j_{m}}, \quad b_{j_{1} \cdots j_{m}} \in \mathbb{C},
\end{aligned}
$$

are such that

$$
(F Q-P)(\underline{x})=\sum_{\left(j_{1}, \ldots, j_{m}\right) \in \mathbb{N}^{m} \backslash E} d_{j_{1} \cdots j_{m}} x_{1}^{j_{1}} \cdots x_{m}^{j_{m}}, \quad d_{j_{1} \cdots j_{m}} \in \mathbb{C}
$$

with $E$ satisfying the inclusion property

$$
\left(j_{1}, \ldots, j_{m}\right) \in E, 0 \leq i_{k} \leq j_{k}, 1 \leq k \leq m \Longrightarrow\left(i_{1}, \ldots, i_{m}\right) \in E .
$$

Equation (1.1) translates to the linear system of equations

$$
\begin{array}{r}
d_{j_{1} \cdots j_{m}}=\sum_{\ell_{1}=0}^{j_{1}} \cdots \sum_{\ell_{m}=0}^{j_{m}} c_{\ell_{1} \cdots \ell_{m}} b_{j_{1}-\ell_{1}, \ldots, j_{m}-\ell_{m}}-a_{j_{1} \cdots j_{m}}=0, \\
\left(j_{1}, \ldots, j_{m}\right) \in E,
\end{array}
$$

where $b_{k_{1} \cdots k_{m}}=0$ for $\left(k_{1}, \ldots, k_{m}\right) \notin N$ and $a_{k_{1} \cdots k_{m}}=0$ for $\left(k_{1}, \ldots, k_{m}\right) \notin M$.

Condition (1.2) takes care of the Padé approximation property, provided $Q(\underline{0}) \neq 0$, namely

$$
\left(F-\frac{P}{Q}\right)(\underline{x})=\sum_{\left(j_{1}, \ldots, j_{m}\right) \in \mathbb{N}^{m} \backslash E} e_{j_{1} \cdots j_{m}} x_{1}^{j_{1}} \cdots x_{m}^{j_{m}}, \quad e_{j_{1} \cdots j_{m}} \in \mathbb{C} .
$$

The linear system (1.3) can be split into two parts: some of the equations serve to compute the numerator and denominator coefficients $a_{j_{1} \cdots j_{m}}$ and $b_{j_{1} \cdots j_{m}}$, while the remaining equations are automatically satisfied by $F Q-P$ for the computed $P$ and $Q$. We refer to the former set of indices $\left(j_{1}, \ldots, j_{m}\right)$ as $C$ and to the latter one as $E \backslash C$. For the class of pseudo-multivariate functions introduced in Definition 1.2 below, very few equations of (1.3) are actually used for the computation of the coefficients. However, in general this is not the case.

One may find a discussion of Definition 1.1 in [6] and more properties of general order multivariate Padé approximants in [3, 4, 5].

Definition 1.2. A multivariate function $F(\underline{x}):=F\left(x_{1}, x_{2}, \ldots, x_{m}\right)$ is said to be pseudo-multivariate if the coefficients of its formal power series

$$
F(\underline{x})=\sum_{j_{1}, \ldots, j_{m}=0}^{\infty} c_{j_{1} \cdots j_{m}} x_{1}^{j_{1}} \cdots x_{m}^{j_{m}}
$$


satisfy

$$
c_{j_{1} \cdots j_{m}}=g\left(j_{1}+\cdots+j_{m}\right), \quad j_{1}, \ldots, j_{m}=0,1, \ldots,
$$

where $g(k)$ is a function of $k$.

A pseudo-multivariate function $F(\underline{x})$ with

$$
\lim _{k \rightarrow \infty}\left|\frac{g(k)}{g(k+1)}\right|=R<\infty
$$

converges in the polydisc centered at the origin with radius $R$. We recall (see [9] for more information) some background on divided differences.

Definition 1.3. The divided difference $f\left[x_{1}, x_{2}, \ldots, x_{n}\right]$ of a function $f(x)$ at distinct $x_{1}, x_{2}, \ldots, x_{n}$ is defined recursively by

$$
\begin{aligned}
& f\left[x_{i}\right]=f\left(x_{i}\right), \quad 1 \leq i \leq n, \\
& f\left[x_{i}, x_{j}\right]=\frac{f\left(x_{j}\right)-f\left(x_{i}\right)}{x_{j}-x_{i}}, \quad 1 \leq i, j \leq n, \\
& f\left[x_{i_{1}}, x_{i_{2}}, \ldots, x_{i_{p}}\right]=\frac{f\left[x_{i_{1}}, x_{i_{2}}, \ldots, x_{i_{p-2}}, x_{i_{p}}\right]-f\left[x_{i_{1}}, x_{i_{2}}, \ldots, x_{i_{p-2}}, x_{i_{p-1}}\right]}{x_{i_{p}}-x_{i_{p-1}}}, \\
& 1 \leq i_{1}, i_{2}, \ldots, i_{p} \leq n ;
\end{aligned}
$$

It is independent of the order of the involved $x_{i}$. If some of the $x_{i}$ coincide, say $x_{i}=x_{i+1}=\cdots=x_{i+k}$ for $1 \leq i, i+k \leq n$, then

$$
f\left[x_{i}, x_{i+1}, \ldots, x_{i+k}\right]:=\lim _{x_{i+1}, \ldots, x_{i+k} \rightarrow x_{i}} f\left[x_{i}, x_{i+1}, \ldots, x_{i+k}\right]=\frac{1}{k !} f^{(k)}\left(x_{i}\right) .
$$

Using this expression the recursive scheme for the computation of the divided differences can be continued for a mixture of distinct and coinciding $x_{i}$.

Note that for distinct $x_{i}$, the divided difference $f\left[x_{1}, x_{2}, \ldots, x_{n}\right]$ can be expressed as a linear combination of the $f\left(x_{i}\right), i=1,2, \ldots, n$, namely,

$$
f\left[x_{1}, x_{2}, \ldots, x_{n}\right]=\sum_{i=1}^{n} \frac{f\left(x_{i}\right)}{\omega_{n}^{\prime}\left(x_{i}\right)},
$$

where

$$
\omega_{n}^{\prime}\left(x_{i}\right)=\prod_{1 \leq j \leq n, j \neq i}\left(x_{i}-x_{j}\right)
$$

is the derivative of

$$
\omega_{n}(x)=\prod_{j=1}^{n}\left(x-x_{j}\right)
$$

at $x=x_{i}$. In addition, the divided difference of the function

$$
f_{n}(x)=x^{m+n-1},
$$

at $x_{1}, x_{2}, \ldots, x_{m}$ is a homogeneous function of degree $n$ in the $m$ variables $x_{1}, x_{2}, \ldots$, $x_{m}$ (see [8] for more details),

$$
f_{n}\left[x_{1}, x_{2}, \ldots, x_{m}\right]=\sum_{i_{1}+i_{2}+\cdots+i_{m}=n} x_{1}^{i_{1}} x_{2}^{i_{2}} \cdots x_{m}^{i_{m}} .
$$

We now discuss how to represent a pseudo-multivariate function as a finite sum of univariate functions. To this end the representation (1.4) is used symbolically, 
meaning that the evaluation $f\left(x_{i}\right)$ at different $x_{i}$ is to be regarded as the use of $f(x)$ for distinct variables $x_{i}$. Coincidence of some $x_{i}$ with $x_{j}$ is to be interpreted as a restriction to the subset of the multidimensional space where $x_{i}=x_{j}$ for some $i \neq j, i, j=1,2, \ldots, m$.

\section{EXPLiCIT CONSTRUCTIONS}

In Theorem 2.1 we show how to use divided differences to obtain a finite sum representation (see [10] for more on this method) of a pseudo-multivariate function $F(\underline{x})$. Afterwards we use this result to obtain explicit formulas for Padé approximants of pseudo-multivariate functions in Theorem 2.2.

Theorem 2.1. Let

$$
F(\underline{x}):=\sum_{n=0}^{\infty} g(n) \sum_{j_{1}+j_{2}+\cdots+j_{m}=n} x_{1}^{j_{1}} x_{2}^{j_{2}} \cdots x_{m}^{j_{m}}
$$

be a pseudo-multivariate function, and let

$$
h(z)=\sum_{k=0}^{\infty} g(k) z^{k} .
$$

If $x_{i} \neq x_{j}$ for all $i, j=1,2, \ldots, m$, we have

$$
F(\underline{x})=\sum_{i=1}^{m} \frac{x_{i}^{m-1}}{\omega_{m}^{\prime}\left(x_{i}\right)} h\left(x_{i}\right),
$$

where $\omega_{m}^{\prime}\left(x_{i}\right)$ is defined by (1.5). If $x_{i}=x_{j}$ for some $i, j=1,2, \ldots, m$, and if $x_{1}, \ldots, x_{r}$ are the distinct variables among the $x_{1}, \ldots, x_{m}$, occurring with multiplicities $s_{1}, \ldots, s_{r}$, respectively, then we have

$$
F(\underline{x})=\sum_{i=1}^{r} \sum_{j=0}^{s_{i}-1} \frac{W_{i}^{\left(s_{i}-j-1\right)}\left(x_{i}\right)}{\left(s_{i}-j-1\right) !} \frac{h^{(j)}\left(x_{i}\right)}{j !},
$$

where

$$
W_{i}(x):=\frac{x^{m-1}}{\prod_{1 \leq j \leq r, j \neq i}\left(x-x_{j}\right)^{s_{j}}}, i=1,2, \ldots, r .
$$

Proof. Let $x_{i} \neq x_{j}$ for $i, j=1,2, \ldots, m$, and let

$$
G(x):=\sum_{n=0}^{\infty} g(n) x^{m+n-1}=\sum_{n=0}^{\infty} g(n) f_{n}(x),
$$

where $f_{n}(x)$ is defined by (1.6). Then by (1.4) and (1.7), and the linearity of the divided difference,

$$
\begin{aligned}
G\left[x_{1}, x_{2}, \ldots, x_{m}\right] & =\sum_{n=0}^{\infty} g(n) f_{n}\left[x_{1}, x_{2}, \ldots, x_{m}\right] \\
& =\sum_{n=0}^{\infty} g(n) \sum_{j_{1}+j_{2}+\cdots+j_{m}=n} x_{1}^{j_{1}} x_{2}^{j_{2}} \cdots x_{m}^{j_{m}} .
\end{aligned}
$$


Also, from (2.3),

$$
\begin{aligned}
G(x) & =\sum_{n=0}^{\infty} g(n) x^{n+m-1} \\
& =x^{m-1} \sum_{n=0}^{\infty} g(n) x^{n} \\
& =x^{m-1} h(x) .
\end{aligned}
$$

By using (2.1), (2.4), and (1.4), we have

$$
\begin{aligned}
F\left(x_{1}, x_{2}, \ldots, x_{m}\right) & =\sum_{n=0}^{\infty} g(n) \sum_{j_{1}+j_{2}+\cdots+j_{m}=n} x_{1}^{j_{1}} x_{2}^{j_{2}} \cdots x_{m}^{j_{m}} \\
& =G\left[x_{1}, x_{2}, \ldots, x_{m}\right] \\
& =\sum_{i=1}^{m} \frac{G\left(x_{i}\right)}{\omega_{m}^{\prime}\left(x_{i}\right)} \\
& =\sum_{i=1}^{m} \frac{x_{i}^{m-1}}{\omega_{m}^{\prime}\left(x_{i}\right)} h\left(x_{i}\right),
\end{aligned}
$$

where $\omega_{m}^{\prime}\left(x_{i}\right)$ is defined by (1.5). Now let $x_{1}, \ldots, x_{r}$ be the distinct variables among all $x_{1}, \ldots, x_{m}$, occurring with multiplicities $s_{1}, \ldots, s_{r}$, respectively, and with $s_{1}+\cdots+s_{r}=m$. Because of the symmetry property of $F\left(x_{1}, x_{2}, \ldots, x_{m}\right)$, we can assume that $x_{1}=x_{r+1}=\cdots=x_{r+s_{1}-1}, x_{2}=x_{r+s_{1}}=\cdots=x_{r+s_{1}+s_{2}-2}$, $\ldots, x_{r}=x_{s_{1}+\cdots+s_{r-1}+2}=\cdots=x_{m}$. Then for $W_{i}\left(x_{i}\right)$ defined by (2.2),

$$
\begin{aligned}
& F\left(x_{1}, x_{2}, \ldots, x_{m}\right) \\
& =F(\overbrace{x_{1}, \ldots, x_{1}}^{s_{1}}, \overbrace{x_{2}, \ldots, x_{2}}^{s_{2}}, \ldots, \overbrace{x_{r}, \ldots, x_{r}}^{s_{r}}) \\
& =\lim _{x_{r+1}, \ldots, x_{r+s_{1}-1} \rightarrow x_{1}} \ldots \lim _{x_{s_{1}+\cdots+s_{r-1}+2, \ldots, x_{m} \rightarrow x_{r}}}\left(\sum_{i=1}^{m} \frac{x_{i}^{m-1} h\left(x_{i}\right)}{\prod_{1 \leq j \leq m, j \neq i}\left(x_{i}-x_{j}\right)}\right) \\
& =\lim _{x_{r+1}, \ldots, x_{r+s_{1}-1} \rightarrow x_{1}} \cdots \lim _{x_{s_{1}+\cdots+s_{r-1}+2}, \ldots, x_{m} \rightarrow x_{r}}\left\{\left(\frac{x_{1}^{m-1} h\left(x_{1}\right)}{\prod_{2 \leq j \leq m}\left(x_{1}-x_{j}\right)}\right.\right. \\
& \left.+\sum_{i=1}^{s_{1}-1} \frac{x_{r+i}^{m-1} h\left(x_{r+i}\right)}{\prod_{1 \leq j \leq m, j \neq r+i}\left(x_{r+i}-x_{j}\right)}\right)+\left(\frac{x_{2}^{m-1} h\left(x_{2}\right)}{\prod_{1 \leq j \leq m, j \neq 2}\left(x_{2}-x_{j}\right)}\right. \\
& \left.+\sum_{i=1}^{s_{2}-1} \frac{x_{r+s_{1}-1+i}^{m-1} h\left(x_{r+s_{1}-1+i}\right)}{\prod_{1 \leq j \leq m, j \neq r+s_{1}-1+i}\left(x_{r+s_{1}-1+i}-x_{j}\right)}\right) \\
& +\cdots+\left(\frac{x_{r}^{m-1} h\left(x_{r}\right)}{\prod_{1 \leq j \leq m, j \neq r}\left(x_{r}-x_{j}\right)}\right. \\
& \left.\left.+\sum_{i=1}^{s_{r}-1} \frac{x_{s_{1}+\cdots+s_{r-1}+1+i}^{m-1} h\left(x_{s_{1}+\cdots+s_{r-1}+1+i}\right)}{\prod_{1 \leq j \leq m, j \neq s_{1}+\cdots+s_{r-1}+1+i}\left(x_{s_{1}+\cdots+s_{r-1}+1+i}-x_{j}\right)}\right)\right\} \\
& =\lim _{x_{r+1}, \ldots, x_{r+s_{1}-1} \rightarrow x_{1}}\left(\frac{W_{1}\left(x_{1}\right) h\left(x_{1}\right)}{\prod_{1 \leq j \leq s_{1}-1}\left(x_{1}-x_{r+j}\right)}\right.
\end{aligned}
$$




$$
\begin{aligned}
& \left.+\sum_{i=1}^{s_{1}-1} \frac{W_{r+i}\left(x_{r+i}\right) h\left(x_{r+i}\right)}{\left(x_{r+i}-x_{1}\right) \prod_{1 \leq j \leq s_{1}-1, j \neq i}\left(x_{r+i}-x_{r+j}\right)}\right) \\
& +\lim _{x_{r+s_{1}}, \ldots, x_{r+s_{1}+s_{2}-2} \rightarrow x_{2}}\left(\frac{W_{2}\left(x_{2}\right) h\left(x_{2}\right)}{\prod_{1 \leq j \leq s_{2}-1}\left(x_{2}-x_{r+s_{1}-1+j}\right)}\right. \\
& \left.+\sum_{i=1}^{s_{2}-1} \frac{W_{r+s_{1}-1+i}\left(x_{r+s_{1}-1+i}\right) h\left(x_{r+s_{1}-1+i}\right)}{\left(x_{r+s_{1}-1+i}-x_{2}\right) \prod_{1 \leq j \leq s_{2}-1, j \neq i}\left(x_{r+s_{1}-1+i}-x_{r+s_{1}-1+j}\right)}\right) \\
& +\cdots+\lim _{x_{s_{1}+\cdots+s_{r-1}+\ldots, x_{m} \rightarrow x_{r}}\left(\frac{W_{r}\left(x_{r}\right) h\left(x_{r}\right)}{\prod_{1 \leq j \leq s_{r}-1}\left(x_{r}-x_{s_{1}+\cdots+s_{r-1}+1+j}\right)}\right.} \\
& \left.+\sum_{i=1}^{s_{r}-1} \frac{W_{s_{1}+\cdots+s_{r-1}+1+i}\left(x_{s_{1}+\cdots+s_{r-1}+1+i}\right) h\left(x_{s_{1}+\cdots+s_{r-1}+1+i}\right)}{\left(x_{s_{1}+\cdots+s_{r-1}+1+i}-x_{r}\right) \prod_{1 \leq j \leq s_{r}-1, j \neq i}\left(x_{s_{1}+\cdots+s_{r-1}+1+i}-x_{s_{1}+\cdots+s_{r-1}+1+j}\right)}\right) .
\end{aligned}
$$

Now if we let

$$
H_{i}(x):=W_{i}(x) h(x), \quad i=1,2, \ldots, r,
$$

then putting it into the equation above, and using the fact that

$$
\lim _{x_{2}, x_{3}, \ldots, x_{r} \rightarrow x_{1}} H\left[x_{1}, x_{2}, \ldots, x_{r}\right]=\frac{1}{(r-1) !} H^{(r-1)}\left(x_{1}\right),
$$

and formula (1.4), we have

$$
\begin{aligned}
F\left(x_{1}, x_{2}, \ldots, x_{m}\right)= & \lim _{x_{r+1}, \ldots, x_{r+s_{1}-1} \rightarrow x_{1}} H_{1}\left[x_{1}, x_{r+1}, \ldots, x_{r+s_{1}-1}\right] \\
& +\lim _{x_{r+s_{1}}, \ldots, x_{r+s_{1}+s_{2}-2} \rightarrow x_{2}} H_{2}\left[x_{2}, x_{r+s_{1}}, \ldots, x_{r+s_{1}+s_{2}-2}\right] \\
& +\cdots+\lim _{x_{s_{1}+\cdots+s_{r-1}+\ldots, x_{m} \rightarrow x_{r}}} H_{r}\left[x_{r}, x_{r+s_{1}+\cdots+s_{r-1}+2}, \ldots, x_{m}\right] \\
& \quad \frac{1}{\left(s_{1}-1\right) !} H_{1}^{\left(s_{1}-1\right)}\left(x_{1}\right)+\frac{1}{\left(s_{2}-1\right) !} H_{2}^{\left(s_{2}-1\right)}\left(x_{2}\right) \\
& +\cdots+\frac{1}{\left(s_{r}-1\right) !} H_{r}^{\left(s_{r}-1\right)}\left(x_{r}\right) \\
= & \sum_{i=1}^{r} \sum_{j=0}^{s_{i}-1} \frac{W_{i}^{\left(s_{i}-j-1\right)}\left(x_{i}\right)}{\left(s_{i}-j-1\right) !} \frac{h^{(j)}\left(x_{i}\right)}{j !} .
\end{aligned}
$$

This proves Theorem 2.1.

Theorem 2.2. Let

$$
F(\underline{x}):=\sum_{k=0}^{\infty} g(k) \sum_{j_{1}+j_{2}+\cdots+j_{m}=k} x_{1}^{j_{1}} x_{2}^{j_{2}} \cdots x_{m}^{j_{m}}
$$

be a pseudo-multivariate function. For $u, v \in \mathbb{N}$, let

$$
\frac{p_{u, v}}{q_{u, v}}(z):=\frac{\sum_{j=0}^{u} \alpha_{j} z^{j}}{\sum_{j=0}^{v} \beta_{j} z^{j}}, \quad \beta_{0}=1
$$

be the $(u, v)$ Padé approximant of the function

$$
h(z):=\sum_{k=0}^{\infty} g(k) z^{k},
$$


and for $\ell=\max \{u, v-1\}$, let

$$
\begin{aligned}
& N:=\left\{\left(j_{1}, j_{2}, \ldots, j_{m}\right): 0 \leq j_{1}, j_{2}, \ldots, j_{m} \leq v\right\} \\
& M:=\left\{\left(j_{1}, j_{2}, \ldots, j_{m}\right): 0 \leq j_{1}, j_{2}, \ldots, j_{m} \leq \ell\right. \\
&\left.j_{1}+j_{2}+\cdots+j_{m} \leq \min \{m \ell, u+(m-1) v\}\right\} \\
& E:=\left\{\left(j_{1}, j_{2}, \ldots, j_{m}\right): 0 \leq j_{1}+j_{2}+\cdots+j_{m} \leq u+v\right\}
\end{aligned}
$$

be index sets in $\mathbb{N}^{m}$. Then if $x_{i} \neq x_{j}$ for all $i, j=1,2, \ldots, m$, the $(M, N)$ general order multivariate Padé approximant to $F(\underline{x})$ on the index set $E$ is

$$
[M / N]_{E}(\underline{x})=\frac{P(\underline{x})}{Q(\underline{x})},
$$

where

$$
\begin{aligned}
& Q(\underline{x})=q_{u, v}\left(x_{1}\right) q_{u, v}\left(x_{2}\right) \cdots q_{u, v}\left(x_{m}\right), \\
& P(\underline{x})=\sum_{i=1}^{m} \frac{x_{i}^{m-1}}{\omega_{m}^{\prime}\left(x_{i}\right)}\left(p_{u, v}\left(x_{i}\right) \prod_{1 \leq k \leq m, k \neq i} q_{u, v}\left(x_{k}\right)\right) .
\end{aligned}
$$

If $x_{i}=x_{j}$ for some $i, j=1,2, \ldots, m$, and if $x_{1}, \ldots, x_{r}$ are the distinct variables among the $x_{1}, \ldots, x_{m}$, occurring with multiplicities $s_{1}, \ldots, s_{r}$, respectively, then $P(x)$ becomes

$$
P(\underline{x})=\sum_{i=1}^{r} \frac{1}{\left(s_{i}-1\right) !}\left(\prod_{1 \leq k \leq r, k \neq i} q_{u, v}^{s_{k}}\left(x_{k}\right)\right)\left(W_{i} p_{u, v} q_{u, v}^{s_{i}-1}\right)^{\left(s_{i}-1\right)}\left(x_{i}\right),
$$

where $W_{i}(x)$ is defined by (2.2).

Proof. From (2.10), we have that

$$
Q(\underline{x})=\prod_{k=1}^{m} q_{u, v}\left(x_{k}\right)=\sum_{\left(j_{1}, j_{2}, \ldots, j_{m}\right) \in N} b_{j_{1} \cdots j_{m}} x_{1}^{j_{1}} x_{2}^{j_{2}} \cdots x_{m}^{j_{m}}, \quad b_{j_{1} \cdots j_{m}} \in \mathbb{C},
$$

where $N$ is defined by (2.7). Now we prove that $P(\underline{x})$ in (2.11) is a polynomial on the index set $M$. For $m \geq 2$,

$$
\begin{aligned}
P(\underline{x})= & \sum_{k=1}^{m} \frac{x_{k}^{m-1}}{\omega_{m}^{\prime}\left(x_{k}\right)}\left(p_{u, v}\left(x_{k}\right) \prod_{1 \leq j \leq m, j \neq k} q_{u, v}\left(x_{j}\right)\right) \\
= & \sum_{k=1}^{m} \frac{x_{k}^{m-1} p_{u, v}\left(x_{k}\right)}{\prod_{1 \leq j \leq m, j \neq k}\left(x_{k}-x_{j}\right)} \prod_{1 \leq j \leq m, j \neq k} q_{u, v}\left(x_{j}\right) \\
= & \frac{1}{\prod_{1 \leq i<j \leq m}\left(x_{i}-x_{j}\right)} \sum_{k=1}^{m}(-1)^{k-1} x_{k}^{m-1} p_{u, v}\left(x_{k}\right) \\
& \quad \times\left(\prod_{1 \leq i<j \leq m, i, j \neq k}\left(x_{i}-x_{j}\right)\right)\left(\prod_{1 \leq j \leq m, j \neq k} q_{u, v}\left(x_{j}\right)\right) .
\end{aligned}
$$


Let

$$
\begin{aligned}
P^{*}(\underline{x}):=\sum_{k=1}^{m}(-1)^{k-1} & x_{k}^{m-1} p_{u, v}\left(x_{k}\right) \\
& \times\left(\prod_{1 \leq i<j \leq m, i, j \neq k}\left(x_{i}-x_{j}\right)\right)\left(\prod_{1 \leq j \leq m, j \neq k} q_{u, v}\left(x_{j}\right)\right) .
\end{aligned}
$$

Then, if $x_{1}=x_{2}$,

$$
\begin{aligned}
P^{*}(\underline{x})= & x_{1}^{m-1} p_{u, v}\left(x_{1}\right)\left(\prod_{2 \leq i<j \leq m}\left(x_{i}-x_{j}\right)\right)\left(\prod_{2 \leq j \leq m} q_{u, v}\left(x_{j}\right)\right) \\
& -x_{2}^{m-1} p_{u, v}\left(x_{2}\right)\left(\prod_{3 \leq j \leq m}\left(x_{1}-x_{j}\right)\right)\left(\prod_{3 \leq i<j \leq m}\left(x_{i}-x_{j}\right)\right) \\
& \quad \times q_{u, v}\left(x_{1}\right)\left(\prod_{3 \leq j \leq m} q_{u, v}\left(x_{j}\right)\right) \\
= & 0,
\end{aligned}
$$

and we have that $\left(x_{1}-x_{2}\right)$ divides $P^{*}(\underline{x})$. As $P(\underline{x})$ is symmetric in its variables, then for $1 \leq i, j \leq m$, if $x_{i}=x_{j}$ for some $i \neq j$,

$$
P^{*}(\underline{x})=0 .
$$

So $\prod_{1 \leq i<j \leq m}\left(x_{i}-x_{j}\right)$ divides $P^{*}(\underline{x})$ and therefore $P(\underline{x})$ is a polynomial of $x_{k}, k=$ $1, \ldots, m$. Now observe that the total degree of $\prod_{1 \leq i<j \leq m}\left(x_{i}-x_{j}\right)$ is $m(m-1) / 2$ and the total degree of $P^{*}(\underline{x})$ is at most

$$
(m-1)+u+\frac{(m-1)(m-2)}{2}+(m-1) v=\frac{m(m-1)}{2}+u+(m-1) v,
$$

so the total degree of $P(\underline{x})$ is at most $u+(m-1) v$. Also observe that the degree of $P^{*}(\underline{x})$ in $x_{1}$ is $\max \{(m-1)+u,(m-2)+v\}$ and the degree of $x_{1}$ in $\prod_{1 \leq i<j \leq m}\left(x_{i}-\right.$ $\left.x_{j}\right)$ is $m-1$, so the degree of $P(\underline{x})$ in $x_{1}$ is $\max \{u, v-1\}$. As $P(\underline{x})$ is symmetric in its variables, the degree of $P(\underline{x})$ in each $x_{k}, k=1, \ldots, m$, is $\max \{u, v-1\}$. This proves that

$$
P(\underline{x}):=\sum_{\left(j_{1}, \ldots, j_{m}\right) \in M} a_{j_{1} \cdots j_{m}} x_{1}^{j_{1}} \cdots x_{m}^{j_{m}}, \quad a_{j_{1} \cdots j_{m}} \in \mathbb{C} .
$$

Now we prove that $[M / N]_{E}(\underline{x})=P(\underline{x}) / Q(\underline{x})$. If $x_{i} \neq x_{j}$ for all $i, j=1,2, \ldots, m$, we have from Theorem 2.1 that,

$$
F(\underline{x})=\sum_{k=1}^{m} \frac{x_{k}^{m-1}}{\omega_{m}^{\prime}\left(x_{k}\right)} h\left(x_{k}\right),
$$

where $h$ is defined by (2.6) and $\omega_{m}^{\prime}$ is defined by (1.5) . Then 


$$
\begin{aligned}
(Q F-P)(\underline{x})= & \left(\prod_{j=1}^{m} q_{u, v}\left(x_{j}\right)\right) \sum_{k=1}^{m} \frac{x_{k}^{m-1}}{\omega_{m}^{\prime}\left(x_{k}\right)} h\left(x_{k}\right) \\
& \quad-\sum_{k=1}^{m} \frac{x_{k}^{m-1}}{\omega_{m}^{\prime}\left(x_{k}\right)}\left(p_{u, v}\left(x_{k}\right) \prod_{1 \leq j \leq m, j \neq k} q_{u, v}\left(x_{j}\right)\right) \\
= & \sum_{k=1}^{m} \frac{x_{k}^{m-1}}{\omega_{m}^{\prime}\left(x_{k}\right)}\left(\prod_{1 \leq j \leq m, j \neq k} q_{u, v}\left(x_{j}\right)\right)\left(q_{u, v}\left(x_{k}\right) h\left(x_{k}\right)-p_{u, v}\left(x_{k}\right)\right) .
\end{aligned}
$$

Recall that $p_{u, v}(z) / q_{u, v}(z)$ defined by (2.5) is the $(u, v)$ Padé approximant to $h(z)$, i.e.

$$
h(z) q_{u, v}(z)-p_{u, v}(z)=\sum_{j \geq u+v+1} \gamma_{j} z^{j}, \quad \gamma_{j} \in \mathbb{C} .
$$

Then

$$
(Q F-P)(\underline{x})=\sum_{k=1}^{m} \frac{x_{k}^{m-1}}{\omega_{m}^{\prime}\left(x_{k}\right)}\left(\prod_{1 \leq j \leq m, j \neq k} q_{u, v}\left(x_{j}\right)\right) \sum_{j \geq u+v+1} \gamma_{j} x_{k}^{j} .
$$

Similar to the proof that $P(\underline{x})$ is a polynomial on index set $M$, we can derive that

$$
\sum_{k=1}^{m} \frac{x_{k}^{m-1}}{\omega_{m}^{\prime}\left(x_{k}\right)}\left(\prod_{1 \leq i \leq m, i \neq k} q_{u, v}\left(x_{i}\right)\right)
$$

is a polynomial of total degree at most $(m-1) v$. Therefore,

$$
\begin{aligned}
(Q F-P)(\underline{x}) & =\sum_{j \geq u+v+1,0 \leq k \leq(m-1) v} \gamma_{j} \sum_{j_{1}+\cdots+j_{m}=j+k} x_{1}^{j_{1}} \cdots x_{m}^{j_{m}} \\
& =\sum_{\left(j_{1}, \ldots, j_{m}\right) \in \mathbb{N}^{m} \backslash E} d_{j_{1} \cdots j_{m}} x_{1}^{j_{1}} \cdots x_{m}^{j_{m}}, \quad d_{j_{1} \cdots j_{m}} \in \mathbb{C},
\end{aligned}
$$

where $E$ is defined by (2.9). This proves Theorem 2.2 for the case $x_{i} \neq x_{j}$ for all $i, j=1,2, \ldots, m$. Now since $Q(\underline{0})=1$ and

$$
\left(F-\frac{P}{Q}\right)(\underline{x})=\sum_{\left(j_{1}, \ldots, j_{m}\right) \in \mathbb{N}^{m} \backslash E} d_{j_{1} \cdots j_{m}}^{*} x_{1}^{j_{1}} \cdots x_{m}^{j_{m}}, \quad d_{j_{1} \cdots j_{m}}^{*} \in \mathbb{C},
$$

we have

$$
=\lim _{x_{r+1}, \ldots, x_{r+s_{1}-1} \rightarrow x_{1}} \cdots \lim _{x_{s_{1}+\cdots+s_{r-1}+2, \ldots, x_{m} \rightarrow x_{r}}}\left(F-\frac{P}{Q}\right)(\underline{x})
$$

On the other hand,

$$
\begin{aligned}
& \lim _{x_{r+1}, \ldots, x_{r+s_{1}-1} \rightarrow x_{1}} \cdots \lim _{x_{s_{1}+\cdots+s_{r-1}+2, \ldots, x_{m} \rightarrow x_{r}}} \frac{P(\underline{x})}{Q(\underline{x})} \\
= & \lim _{x_{r+1}, \ldots, x_{r+s_{1}-1} \rightarrow x_{1}} \cdots \lim _{x_{s_{1}+\cdots+s_{r-1}+2, \ldots, x_{m} \rightarrow x_{r}}} \frac{1}{Q(\underline{x})}
\end{aligned}
$$




$$
\begin{aligned}
& \times \sum_{i=1}^{m} \frac{x_{i}^{m-1}}{\omega_{m}^{\prime}\left(x_{i}\right)}\left(p_{u, v}\left(x_{i}\right) \prod_{1 \leq k \leq m, k \neq i} q_{u, v}\left(x_{k}\right)\right) \\
& =\frac{1}{q_{u, v}^{s_{1}}\left(x_{1}\right) q_{u, v}^{s_{2}}\left(x_{2}\right) \cdots q_{u, v}^{s_{r}}\left(x_{r}\right)} \\
& \times \lim _{x_{r+1}, \ldots, x_{r+s_{1}-1} \rightarrow x_{1}} \cdots \lim _{x_{s_{1}+\cdots+s_{r-1}+2}, \ldots, x_{m} \rightarrow x_{r}} \\
& \times \sum_{i=1}^{m} \frac{x_{i}^{m-1}}{\omega_{m}^{\prime}\left(x_{i}\right)}\left(p_{u, v}\left(x_{i}\right) \prod_{1 \leq k \leq m, k \neq i} q_{u, v}\left(x_{k}\right)\right) \\
& =\frac{q_{u, v}^{s_{2}}\left(x_{2}\right) \cdots q_{u, v}^{s_{r}}\left(x_{r}\right)}{q_{u, v}^{s_{1}}\left(x_{1}\right) q_{u, v}^{s_{2}}\left(x_{2}\right) \cdots q_{u, v}^{s_{r}}\left(x_{r}\right)} \\
& \times \lim _{x_{r+1}, \ldots, x_{r+s_{1}-1} \rightarrow x_{1}}\left(\frac{W_{1}\left(x_{1}\right) p_{u, v}\left(x_{1}\right) q_{u, v}^{s_{1}-1}\left(x_{1}\right)}{\prod_{1 \leq j \leq s_{1}-1}\left(x_{1}-x_{r+j}\right)}\right. \\
& \left.+\sum_{i=1}^{s_{1}-1} \frac{W_{r+i}\left(x_{r+i}\right) p_{u, v}\left(x_{r+i}\right) q_{u, v}^{s_{1}-1}\left(x_{r+i}\right)}{\left(x_{r+i}-x_{1}\right) \prod_{1 \leq j \leq s_{1}-1, j \neq i}\left(x_{r+i}-x_{r+j}\right)}\right) \\
& +\cdots+\frac{q_{u, v}^{s_{1}}\left(x_{1}\right) \cdots q_{u, v}^{s_{r}-1}\left(x_{r-1}\right)}{q_{u, v}^{s_{1}}\left(x_{1}\right) q_{u, v}^{s_{2}}\left(x_{2}\right) \cdots q_{u, v}^{s_{r}}\left(x_{r}\right)} \\
& \times \lim _{x_{s_{1}+\cdots+s_{r-1}+2, \ldots, x_{m} \rightarrow x_{r}}}\left(\frac{W_{r}\left(x_{r}\right) p_{u, v}\left(x_{r}\right) q_{u, v}^{s_{r}-1}\left(x_{r}\right)}{\prod_{1 \leq j \leq s_{r}-1}\left(x_{r}-x_{s_{1}+\cdots+s_{r-1}+1+j}\right)}\right. \\
& +\sum_{i=1}^{s_{r}-1}\left[\frac{W_{s_{1}+\cdots+s_{r-1}+1+i}\left(x_{s_{1}+\cdots+s_{r-1}+1+i}\right)}{x_{s_{1}+\cdots+s_{r-1}+1+i}-x_{r}}\right. \\
& \left.\left.\times \frac{p_{u, v}\left(x_{s_{1}+\cdots+s_{r-1}+1+i}\right) q_{u, v}^{s_{r}-1}\left(x_{s_{1}+\cdots+s_{r-1}+1+i}\right)}{\prod_{1 \leq j \leq s_{r}-1, j \neq i}\left(x_{s_{1}+\cdots+s_{r-1}+1+i}-x_{s_{1}+\cdots+s_{r-1}+1+j}\right)}\right]\right) .
\end{aligned}
$$

If we let

$$
H_{i}^{*}(x):=W_{i}(x) p_{u, v}(x) q_{u, v}^{s_{i}-1}(x), \quad i=1,2, \ldots, r
$$

then

$$
\begin{aligned}
& \lim _{x_{r+1}, \ldots, x_{r+s_{1}-1} \rightarrow x_{1}} \ldots \lim _{x_{s_{1}+\cdots+s_{r-1}+2}, \ldots, x_{m} \rightarrow x_{r}} \frac{P(\underline{x})}{Q(\underline{x})} \\
& =\frac{q_{u, v}^{s_{2}}\left(x_{2}\right) \cdots q_{u, v}^{s_{r}}\left(x_{r}\right)}{q_{u, v}^{s_{1}}\left(x_{1}\right) q_{u, v}^{s_{2}}\left(x_{2}\right) \cdots q_{u, v}^{s_{r}}\left(x_{r}\right)} \\
& \times \lim _{x_{r+1}, \ldots, x_{r+s_{1}-1} \rightarrow x_{1}} H_{1}^{*}\left[x_{1}, x_{r+1}, \ldots, x_{r+s_{1}-1}\right] \\
& +\cdots+\frac{q_{u, v}^{s_{1}}\left(x_{1}\right) \cdots q_{u, v}^{s_{r}-1}\left(x_{r-1}\right)}{q_{u, v}^{s_{1}}\left(x_{1}\right) q_{u, v}^{s_{2}}\left(x_{2}\right) \cdots q_{u, v}^{s_{r}}\left(x_{r}\right)} \\
& \times \lim _{x_{s_{1}+\cdots+s_{r-1}+2, \ldots, x_{m} \rightarrow x_{r}}} H_{r}^{*}\left[x_{r}, x_{s_{1}+\cdots+s_{r-1}+2}, \ldots, x_{m}\right] \\
& =\frac{1}{q_{u, v}^{s_{1}}\left(x_{1}\right) q_{u, v}^{s_{2}}\left(x_{2}\right) \cdots q_{u, v}^{s_{r}}\left(x_{r}\right)} \\
& \times\left(\frac{q_{u, v}^{s_{2}}\left(x_{2}\right) \cdots q_{u, v}^{s_{r}}\left(x_{r}\right)}{\left(s_{1}-1\right) !}\left(H_{1}^{*}\right)^{\left(s_{1}-1\right)}\left(x_{1}\right)\right.
\end{aligned}
$$




$$
\begin{aligned}
& \left.+\cdots+\frac{q_{u, v}^{s_{1}}\left(x_{1}\right) \cdots q_{u, v}^{s_{r}-1}\left(x_{r-1}\right)}{\left(s_{r}-1\right) !}\left(H_{r}^{*}\right)^{\left(s_{r}-1\right)}\left(x_{r}\right)\right) \\
= & \frac{1}{q_{u, v}^{s_{1}}\left(x_{1}\right) q_{u, v}^{s_{2}}\left(x_{2}\right) \cdots q_{u, v}^{s_{r}}\left(x_{r}\right)} \\
& \times \sum_{i=1}^{r} \frac{1}{\left(s_{i}-1\right) !}\left(\prod_{1 \leq k \leq r, k \neq i} q_{u, v}^{s_{k}}\left(x_{k}\right)\right)\left(W_{i} p_{u, v} q_{u, v}^{s_{i}-1}\right)^{\left(s_{i}-1\right)}\left(x_{i}\right) .
\end{aligned}
$$

So if $x_{1}, \ldots, x_{r}$ are the distinct variables among all $x_{1}, \ldots, x_{m}$, occurring with multiplicities $s_{1}, \ldots, s_{r}$, respectively, then

$$
Q(\underline{x})=q_{u, v}^{s_{1}}\left(x_{1}\right) q_{u, v}^{s_{2}}\left(x_{2}\right) \cdots q_{u, v}^{s_{r}}\left(x_{r}\right),
$$

which is (2.10), and

$$
P(\underline{x})=\sum_{i=1}^{r} \frac{1}{\left(s_{i}-1\right) !}\left(\prod_{1 \leq k \leq r, k \neq i} q_{u, v}^{s_{k}}\left(x_{k}\right)\right)\left(W_{i} p_{u, v} q_{u, v}^{s_{i}-1}\right)^{\left(s_{i}-1\right)}\left(x_{i}\right),
$$

which is the limiting case of $P(\underline{x})$ in (2.11). This proves Theorem 2.2.

Remark. It is easy to see that $P(\underline{x}) / Q(\underline{x})$ is irreducible, as $p_{u, v}(z) / q_{u, v}(z)$ is irreducible.

\section{Properties of pseudo-multivariate Padé approximants}

The univariate Padé approximant satisfies a consistency property, meaning that when the given function $h$ is itself rational, then it is reconstructed by $p_{u, v} / q_{u, v}$ when $u$ and $v$ are chosen large enough. This consistency property holds mainly because of the unicity of the irreducible form of $p_{u, v} / q_{u, v}$. For a general order multivariate Padé approximant this is not necessarily the case, because of the possible nonunicity of the irreducible form of the approximant. However, similar to the properties proved in our earlier paper [6], the general order multivariate Padé approximants constructed in Theorem 2.2 in this paper have many nice properties. We prove the consistency and normality properties of these approximants, the latter meaning that if the univariate $(u, v)$ Padé approximant $p_{u, v} / q_{u, v}$ to the function $h$ appears only once in the Padé table, then so does its general multivariate counterpart constructed here. At the end of this section, we also present a truncation error upperbound. The properties proved in [6] constitute the special bivariate case of the properties in this section.

Theorem 3.1. Let $u, v, M, N, E$ be defined as in Theorem 2.2. If the pseudomultivariate function $F(\underline{x})$ is a rational function, i.e. if $F(\underline{x})$ has the irreducible form

$$
F(\underline{x}):=\frac{R(\underline{x})}{S(\underline{x})}=\frac{\sum_{\left(j_{1}, j_{2}, \ldots, j_{m}\right) \in M} r_{j_{1} \cdots j_{m}} x_{1}^{j_{1}} x_{2}^{j_{2}} \cdots x_{m}^{j_{m}}}{\sum_{\left(j_{1}, j_{2}, \ldots, j_{m}\right) \in N} s_{j_{1} \cdots j_{m}} x_{1}^{j_{1}} x_{2}^{j_{2}} \cdots x_{m}^{j_{m}}},
$$

with $r_{j_{1} \cdots j_{m}}, s_{j_{1} \cdots j_{m}} \in \mathbb{C}$ and $S(\underline{0}) \neq 0$, then the $(M, N)$ general order multivariate Padé approximant to $F(\underline{x})$ on the index set $E$, constructed in Theorem 2.2, satisfies

$$
\frac{P(\underline{x})}{Q(\underline{x})}=F(\underline{x})
$$

for large enough $u, v \in \mathbb{N}$. 
Proof. As $F$ is a pseudo-multivariate function, we have

$$
F(\underline{x})=\sum_{n=0}^{\infty} g(n) \sum_{j_{1}+j_{2}+\cdots+j_{m}=n} x_{1}^{j_{1}} x_{2}^{j_{2}} \cdots x_{m}^{j_{m}}
$$

with

$$
h(z)=\sum_{k=0}^{\infty} g(k) z^{k} .
$$

Then $h(z)=F(z, 0, \ldots, 0)=F(0, z, 0, \ldots, 0)=\cdots=F(0, \cdots, 0, z)$ and hence

$$
h(z)=\frac{R(z, 0, \ldots, 0)}{S(z, 0, \ldots, 0)}=\cdots=\frac{R(0, \ldots, 0, z)}{S(0, \ldots, 0, z)} .
$$

From the consistency property of the univariate Padé aproximant, we have for large enough $u, v \in \mathbb{N}, p_{u, v}(z)=R(z, 0, \ldots, 0)=\ldots=R(0, \ldots, 0, z)$ and $q_{u, v}(z)=$ $S(z, 0, \ldots, 0)=\cdots=S(0, \ldots, 0, z)$ after a suitable normalization of $q_{u, v}(z)$. So if $x_{i} \neq x_{j}$ for all $i, j=1,2, \ldots, m$, then from Theorem 2.1 and Theorem 2.2, we have

$$
\begin{aligned}
\frac{P(\underline{x})}{Q(\underline{x})} & =\frac{1}{q_{u, v}\left(x_{1}\right) \cdots q_{u, v}\left(x_{m}\right)} \sum_{i=1}^{m} \frac{x_{i}^{m-1}}{\omega_{m}^{\prime}\left(x_{i}\right)}\left(p_{u, v}\left(x_{i}\right) \prod_{1 \leq k \leq m, k \neq i} q_{u, v}\left(x_{k}\right)\right) \\
& =\sum_{i=1}^{m} \frac{x_{i}^{m-1}}{\omega_{m}^{\prime}\left(x_{i}\right)} \frac{p_{u, v}\left(x_{i}\right)}{q_{u, v}\left(x_{i}\right)} \\
& =\sum_{i=1}^{m} \frac{x_{i}^{m-1}}{\omega_{m}^{\prime}\left(x_{i}\right)} h\left(x_{i}\right) \\
& =F(\underline{x}),
\end{aligned}
$$

Now if $x_{i}=x_{j}$ for some $i, j=1,2, \ldots, m$, we denote the distinct variables among the $x_{1}, \ldots, x_{m}$ by $x_{1}, \ldots, x_{r}$, which occur with multiplicities $s_{1}, \ldots, s_{r}$, respectively. By Theorem 2.2, the $(M, N)$ general order multivariate Padé approximant to $F(\underline{x})$ on the index set $E$ is the limiting case,

$$
\begin{aligned}
& \frac{P(\underline{x})}{Q(\underline{x})}=\lim _{x_{r+1}, \ldots, x_{r+s_{1}-1} \rightarrow x_{1}} \ldots \lim _{x_{s_{1}+\cdots+s_{r-1}+2}, \ldots, x_{m} \rightarrow x_{r}}\left\{\frac{1}{q_{u, v}\left(x_{1}\right) \cdots q_{u, v}\left(x_{m}\right)}\right. \\
& \left.\times \sum_{i=1}^{m} \frac{x_{i}^{m-1}}{\omega_{m}^{\prime}\left(x_{i}\right)}\left(p_{u, v}\left(x_{i}\right) \prod_{1 \leq k \leq m, k \neq i} q_{u, v}\left(x_{k}\right)\right)\right\} \\
& =\lim _{x_{r+1}, \ldots, x_{r+s_{1}-1} \rightarrow x_{1}} \ldots \lim _{x_{s_{1}+\cdots+s_{r-1}+2, \ldots, x_{m} \rightarrow x_{r}}} \sum_{i=1}^{m} \frac{x_{i}^{m-1}}{\omega_{m}^{\prime}\left(x_{i}\right)} \frac{p_{u, v}\left(x_{i}\right)}{q_{u, v}\left(x_{i}\right)} \\
& =\lim _{x_{r+1}, \ldots, x_{r+s_{1}-1} \rightarrow x_{1}} \ldots \lim _{x_{s_{1}+\cdots+s_{r-1}+2, \ldots, x_{m} \rightarrow x_{r}}} \sum_{i=1}^{m} \frac{x_{i}^{m-1}}{\omega_{m}^{\prime}\left(x_{i}\right)} h\left(x_{i}\right) \\
& =\lim _{x_{r+1}, \ldots, x_{r+s_{1}-1} \rightarrow x_{1}} \cdots \lim _{x_{s_{1}+\cdots+s_{r-1}+2, \ldots, x_{m} \rightarrow x_{r}}} F(\underline{x}) \\
& =F(\underline{x}),
\end{aligned}
$$

This completes the proof of Theorem 3.1.

Theorem 3.2. For $u, v \in \mathbb{N}$, let $M, N, E, F(\underline{x})$ and $h(z)$ be defined as in Theorem 2.1 and Theorem 2.2. If the $(u, v)$ Padé approximant $p_{u, v}(z) / q_{u, v}(z)$ to $h(z)$ is 
normal, then the $(M, N)$ Padé approximant $P(\underline{x}) / Q(\underline{x})$ to $F(\underline{x})$ on the index set $E$ given in Theorem 2.2, is also normal.

Proof. If the $(u, v)$ Padé approximant to $h(z)$,

$$
\frac{p_{u, v}}{q_{u, v}}(z)=\frac{\sum_{j=0}^{u} \alpha_{j} z^{j}}{\sum_{j=0}^{v} \beta_{j} z^{j}}, \quad \beta_{0}=1
$$

is normal for $u, v \in \mathbb{N}$, then

$$
\alpha_{u} \neq 0, \quad \beta_{v} \neq 0
$$

and

$$
\left(h q_{u, v}-p_{u, v}\right)(z)=\sum_{j \geq u+v+1} \gamma_{j} z^{j}
$$

with

$$
\gamma_{u+v+1} \neq 0
$$

To prove the normality of the multivariate Padé approximant it suffices to prove it for the case that if $x_{i} \neq x_{j}$ for all $i, j=1,2, \ldots, m$. The $(M, N)$ Padé approximant to $F(\underline{x})$ on the set $E$ is $P(\underline{x}) / Q(\underline{x})$, where

$$
Q(\underline{x})=\prod_{k=1}^{m} q_{u, v}\left(x_{k}\right)=\sum_{\left(j_{1}, j_{2}, \ldots, j_{m}\right) \in N} b_{j_{1} \cdots j_{m}} x_{1}^{j_{1}} x_{2}^{j_{2}} \cdots x_{m}^{j_{m}}, \quad b_{j_{1} \cdots j_{m}} \in \mathbb{C},
$$

and

$$
\begin{aligned}
P(\underline{x}) & =\sum_{i=1}^{m} \frac{x_{i}^{m-1}}{\omega_{m}^{\prime}\left(x_{i}\right)}\left(p_{u, v}\left(x_{i}\right) \prod_{1 \leq k \leq m, k \neq i} q_{u, v}\left(x_{k}\right)\right) \\
& =\sum_{\left(j_{1}, j_{2}, \ldots, j_{m}\right) \in M} a_{j_{1} \cdots j_{m}} x_{1}^{j_{1}} x_{2}^{j_{2}} \cdots x_{m}^{j_{m}}, a_{j_{1} \cdots j_{m}} \in \mathbb{C},
\end{aligned}
$$

with

$$
\begin{aligned}
b_{0 \cdots 0} & =\left(\beta_{0}\right)^{m}=1 \\
b_{v \cdots v} & =\left(\beta_{v}\right)^{m} \neq 0 \\
b_{0 \cdots 0 v} & =\cdots=b_{v 0 \cdots 0}=\beta_{v}\left(\beta_{0}\right)^{m-1} \neq 0
\end{aligned}
$$

and

Now

$$
a_{u 0 \cdots 0}=\cdots=a_{0 \cdots 0 u}=\alpha_{u}\left(\beta_{0}\right)^{m-1} \neq 0 .
$$

$$
\begin{aligned}
(F Q-P)(\underline{x})= & \left(\prod_{k=1}^{m} q_{u, v}\left(x_{k}\right)\right) \sum_{i=1}^{m} \frac{x_{i}^{m-1}}{\omega_{m}^{\prime}\left(x_{i}\right)} h\left(x_{i}\right) \\
& -\sum_{i=1}^{m} \frac{x_{i}^{m-1}}{\omega_{m}^{\prime}\left(x_{i}\right)}\left(p_{u, v}\left(x_{i}\right) \prod_{1 \leq k \leq m, k \neq i} q_{u, v}\left(x_{k}\right)\right) \\
= & \sum_{i=1}^{m} \frac{x_{i}^{m-1}}{\omega_{m}^{\prime}\left(x_{i}\right)}\left(\prod_{1 \leq k \leq m, k \neq i} q_{u, v}\left(x_{k}\right)\right)\left(h\left(x_{i}\right) q_{u, v}\left(x_{i}\right)-p_{u, v}\left(x_{i}\right)\right) \\
= & \sum_{i=1}^{m} \frac{x_{i}^{m-1}}{\omega_{m}^{\prime}\left(x_{i}\right)}\left(\prod_{1 \leq k \leq m, k \neq i} q_{u, v}\left(x_{k}\right)\right) \sum_{j \geq u+v+1} \gamma_{j} x_{i}^{j} .
\end{aligned}
$$


So if

$$
(F Q-P)(\underline{x})=\sum_{\left(j_{1}, j_{2}, \ldots, j_{m}\right) \in E} d_{j_{1} \cdots j_{m}} x_{1}^{j_{1}} x_{2}^{j_{2}} \cdots x_{m}^{j_{m}}, \quad d_{j_{1} \cdots j_{m}} \in \mathbb{C},
$$

then for $j_{1}+\cdots+j_{m}=u+v+1$,

$$
d_{j_{1} \cdots j_{m}}=\left(\beta_{0}\right)^{m-1} \gamma_{u+v+1}=\gamma_{u+v+1} \neq 0 .
$$

Now assume that for either $u^{\prime} \neq u$ or $v^{\prime} \neq v, \ell^{\prime}=\max \left\{u^{\prime}, v^{\prime}-1\right\}$ with

$$
\begin{aligned}
N^{\prime}=\left\{\left(j_{1}, j_{2}, \ldots, j_{m}\right): 0 \leq\right. & \left.j_{1}, j_{2}, \ldots, j_{m} \leq v^{\prime}\right\} \\
M^{\prime}=\left\{\left(j_{1}, j_{2}, \ldots, j_{m}\right): 0 \leq\right. & j_{1}, j_{2}, \ldots, j_{m} \leq \ell^{\prime}, j_{1}+j_{2} \\
& \left.+\cdots+j_{m} \leq u^{\prime}+(m-1) v^{\prime}\right\}, \\
E^{\prime}=\left\{\left(j_{1}, j_{2}, \ldots, j_{m}\right): 0 \leq\right. & \left.j_{1}+j_{2}+\cdots+j_{m} \leq u^{\prime}+v^{\prime}\right\}
\end{aligned}
$$

the general order multivariate Padé approximant $\left[M^{\prime} / N^{\prime}\right]_{E^{\prime}}$ for $F(\underline{x})$ on the set $E^{\prime}$ equals the same rational function $P / Q$. Since $\alpha_{u}=a_{u 0 \cdots 0} \neq 0$ and $\beta_{v}=b_{v 0 \cdots 0} \neq 0$, this is only possible for $u^{\prime} \geq u$ and $v^{\prime} \geq v$. Hence $u^{\prime}+v^{\prime}+1 \geq u+v+1$. The fact that $\gamma_{u+v+1} \neq 0$ reduces the occurrence of nonnormality to $u^{\prime}+v^{\prime}+1 \leq u+v+1$. Hence $u^{\prime}+v^{\prime}+1=u+v+1$. In combination with $u^{\prime} \geq u$ and $v^{\prime} \geq v$ this leads to $u^{\prime}=u$ and $v^{\prime}=v$. Since the latter is a contradiction with our assumption that either $u^{\prime} \neq u$ or $v^{\prime} \neq v$, normality must hold.

Theorem 3.3. Let $M, N, E, F(\underline{x})$ and $h(z)$ be defined as in Theorem 2.1 and Theorem 2.2. Let $p_{u, v}(z) / q_{u, v}(z)$ be the $(u, v)$ Padé approximant to $h(z)$ and $P(\underline{x}) / Q(\underline{x})$ the $(M, N)$ Padé approximant, constructed in Theorem 2.2 to $F(\underline{x})$ on E. Let the function $h(z)$ be analytic in a disk $B(0, \rho)$ centered at the origin with radius $\rho>\max _{1 \leq i \leq m}\left|x_{i}\right|$. Then, if $x_{i} \neq x_{j}$ for all $i \neq j, i, j=1,2, \ldots, m$,

$$
\left|\left(F-\frac{P}{Q}\right)(\underline{x})\right| \leq \frac{\sup _{\xi \in B(0, \rho)}\left|\left(h q_{u, v}\right)^{(u+v+1)}(\xi)\right|}{(u+v+1) !} \sum_{i=1}^{m}\left|\frac{x_{i}^{u+v+m}}{\omega_{m}^{\prime}\left(x_{i}\right) q_{u, v}\left(x_{i}\right)}\right| .
$$

If $x_{i}=x_{j}$ for some $i \neq j, i, j=1,2, \ldots, m$, we assume that $x_{1}, \ldots, x_{r}$ are the distinct variables among the $x_{1}, \ldots, x_{m}$, occurring with multiplicities $s_{1}, \ldots, s_{r}$, respectively, then

$$
\begin{aligned}
& \left|\left(F-\frac{P}{Q}\right)(\underline{x})\right| \\
& \quad \leq \frac{\sup _{\xi \in B(0, \rho)}\left|\left(h q_{u, v}\right)^{(u+v+1)}(\xi)\right|}{(u+v+1) !} \sum_{i=1}^{r} \frac{1}{\left(s_{i}-1\right) !}\left|\left(\frac{W_{i}\left(x_{i}\right) x_{i}^{u+v+1}}{q_{u, v}\left(x_{i}\right)}\right)^{\left(s_{i}-1\right)}\right| .
\end{aligned}
$$

Proof. For the univariate function $h(z)$ and its $(u, v)$ Padé approximant $p_{m, n} / q_{m, n}$ we know that

$$
\left|\left(h q_{u, v}-p_{u, v}\right)(z)\right| \leq \sup _{\xi_{z} \in[0, z]}\left|\left(h q_{u, v}\right)^{(u+v+1)}\left(\xi_{z}\right)\right| \frac{|z|^{u+v+1}}{(u+v+1) !},
$$

If $x_{i} \neq x_{j}$ for all $i, j=1,2, \ldots, m$, then from (3.1) and (3.2), 


$$
\begin{aligned}
\mid(F Q & -P)(\underline{x})|=| \sum_{i=1}^{m} \frac{x_{i}^{m-1}}{\omega_{m}^{\prime}\left(x_{i}\right)}\left(\prod_{1 \leq k \leq m, k \neq i} q_{u, v}\left(x_{k}\right)\right)\left(h\left(x_{i}\right) q_{u, v}\left(x_{i}\right)-p_{u, v}\left(x_{i}\right)\right) \mid \\
& \leq \sum_{i=1}^{m}\left|\frac{x_{i}^{m-1}}{\omega_{m}^{\prime}\left(x_{i}\right)}\right|\left|\prod_{1 \leq k \leq m, k \neq i} q_{u, v}\left(x_{k}\right)\right|\left|h\left(x_{i}\right) q_{u, v}\left(x_{i}\right)-p_{u, v}\left(x_{i}\right)\right| \\
& \leq \sum_{i=1}^{m} \frac{\left|x_{i}\right|^{u+v+m}}{\left|\omega_{m}^{\prime}\left(x_{i}\right)\right|(u+v+1) !}\left|\prod_{1 \leq k \leq m, k \neq i} q_{u, v}\left(x_{k}\right)\right| \sup _{\xi_{i} \in\left[0, x_{i}\right]}\left|\left(h q_{u, v}\right)^{(u+v+1)}\left(\xi_{i}\right)\right| .
\end{aligned}
$$

So

$$
\left|\left(F-\frac{P}{Q}\right)(\underline{x})\right| \leq \frac{\sup _{\xi \in B(0, \rho)}\left|\left(h q_{u, v}\right)^{(u+v+1)}(\xi)\right|}{(u+v+1) !} \sum_{i=1}^{m}\left|\frac{x_{i}^{u+v+m}}{\omega_{m}^{\prime}\left(x_{i}\right) q_{u, v}\left(x_{i}\right)}\right| .
$$

Now if $x_{i}=x_{j}$ for some $i, j=1,2, \ldots, m$ and if $x_{1}, \ldots, x_{r}$ are the distinct variables among the $x_{1}, \ldots, x_{m}$, occurring with multiplicities $s_{1}, \ldots, s_{r}$, respectively, then by Theorem 2.2 the $(M, N)$ general order multivariate Padé approximant to $F(\underline{x})$ on the index set $E$ is the limiting case. So

$$
\begin{aligned}
& \lim _{x_{r+1}, \ldots, x_{r+s_{1}-1} \rightarrow x_{1}} \cdots \lim _{x_{s_{1}+\cdots+s_{r-1}+2, \ldots, x_{m} \rightarrow x_{r}}}\left(F-\frac{P}{Q}\right)(\underline{x}) \\
\leq & \frac{\sup _{\xi \in B(0, \rho)}\left|\left(h q_{u, v}\right)^{(u+v+1)}(\xi)\right|}{(u+v+1) !} \\
& \times \lim _{x_{r+1}, \ldots, x_{r+s_{1}-1} \rightarrow x_{1}} \cdots \lim _{x_{s_{1}+\cdots+s_{r-1}+2, \ldots, x_{m} \rightarrow x_{r}}} \sum_{i=1}^{m}\left|\frac{x_{i}^{m-1}}{\omega_{m}^{\prime}\left(x_{i}\right)} \frac{x_{i}^{u+v+1}}{q_{u, v}\left(x_{i}\right)}\right| .
\end{aligned}
$$

By using the same strategy as that in the proofs of Theorem 2.1 and Theorem 2.2, we find

$$
\begin{aligned}
& \lim _{x_{r+1}, \ldots, x_{r+s_{1}-1} \rightarrow x_{1}} \ldots \lim _{x_{s_{1}+\cdots+s_{r-1}+2, \ldots, x_{m} \rightarrow x_{r}}} \sum_{i=1}^{m}\left|\frac{x_{i}^{m-1}}{\omega_{m}^{\prime}\left(x_{i}\right)} \frac{x_{i}^{u+v+1}}{q_{u, v}\left(x_{i}\right)}\right| \\
= & \sum_{i=1}^{r} \frac{1}{\left(s_{i}-1\right) !}\left|\left(\frac{W_{i}\left(x_{i}\right) x_{i}^{u+v+1}}{q_{u, v}\left(x_{i}\right)}\right)^{\left(s_{i}-1\right)}\right| .
\end{aligned}
$$

Therefore,

$$
\begin{aligned}
& \left|\left(F-\frac{P}{Q}\right)(\underline{x})\right| \\
& \quad \leq \frac{\sup _{\xi \in B(0, \rho)}\left|\left(h q_{u, v}\right)^{(u+v+1)}(\xi)\right|}{(u+v+1) !} \sum_{i=1}^{r} \frac{1}{\left(s_{i}-1\right) !}\left|\left(\frac{W_{i}\left(x_{i}\right) x_{i}^{u+v+1}}{q_{u, v}\left(x_{i}\right)}\right)^{\left(s_{i}-1\right)}\right| .
\end{aligned}
$$

This completes the proof of Theorem 3.3.

\section{EXAMPLES}

Throughout this section, we let $M, N$ and $E$ be defined as in Theorem 2.2. We first see some examples of finding the explicit Padé approximants for some pseudomultivariate functions of which the one variable projections have general explicit formulas of Padé approximants. 
Example 4.1. A multivariate form of the exponential function is

$$
E(\underline{x})=\sum_{j_{1}, j_{2}, \ldots, j_{m}=0}^{\infty} \frac{x_{1}^{j_{1}} x_{2}^{j_{2}} \cdots x_{m}^{j_{m}}}{\left(j_{1}+j_{2}+\cdots+j_{m}\right) !} .
$$

It is a pseudo-multivariate function with

$$
h(z)=\exp (z)=\sum_{k=0}^{\infty} \frac{z^{k}}{k !} .
$$

From [2], we have that the $(u, v)$ Padé approximant to $h(z)$ is

$$
\frac{p_{u, v}}{q_{u, v}}(z)=\frac{{ }_{1} F_{1}(-u ;-u-v ; z)}{{ }_{1} F_{1}(-v ;-u-v ;-z)},
$$

and then, by Theorem 2.2, if $x_{i} \neq x_{j}$ for all $i, j=1,2, \ldots, m$, the $(M, N)$ general order multivariate Padé approximant to $E(\underline{x})$ is $P(\underline{x}) / Q(\underline{x})$, where

$$
\begin{aligned}
Q(\underline{x}) & =\prod_{j=1}^{m}{ }_{1} F_{1}\left(-v ;-u-v ;-x_{j}\right) \\
& =\sum_{j_{1}, \ldots, j_{m}=1}^{m} \frac{(-1)^{j_{1}+\cdots+j_{m}}(-v)_{j_{1}} \cdots(-v)_{j_{m}}}{(-u-v)_{j_{1}} \cdots(-u-v)_{j_{m}}} x^{j_{1}} \cdots x_{m}^{j_{m}},
\end{aligned}
$$

and

$$
P(\underline{x})=\sum_{i=1}^{m} \frac{x_{i}^{m-1}}{\omega_{m}^{\prime}\left(x_{i}\right)}{ }_{1} F_{1}\left(-u ;-u-v ; x_{i}\right) \prod_{1 \leq k \leq m, k \neq i}{ }_{1} F_{1}\left(-v ;-u-v ; x_{k}\right) .
$$

For pseudo-multivariate functions of which the one variable projection doesn't have an explicit formula for its Padé approximants, we can use software to compute the $(M, N)$ general order multivariate Padé approximant for given $M, N$. Below is an example of a short procedure in Maple to compute the $(M, N)$ general order multivariate Padé approximant for trivariate pseudo-multivariate functions.

$\mathrm{mpa}(\mathrm{f}, \mathrm{x}, \mathrm{u}, \mathrm{v})-f$ is the one variable projection of the pseudo-multivariate function $F, x$ is the variable of $f$ and $u, v$ are nonnegative integers. The procedure computes the $(M, N)$ general order multivariate Padé approximant to $F(x, y, z)$ on the set $E$, where $M, N$, and $E$ are defined in Theorem 2.2.

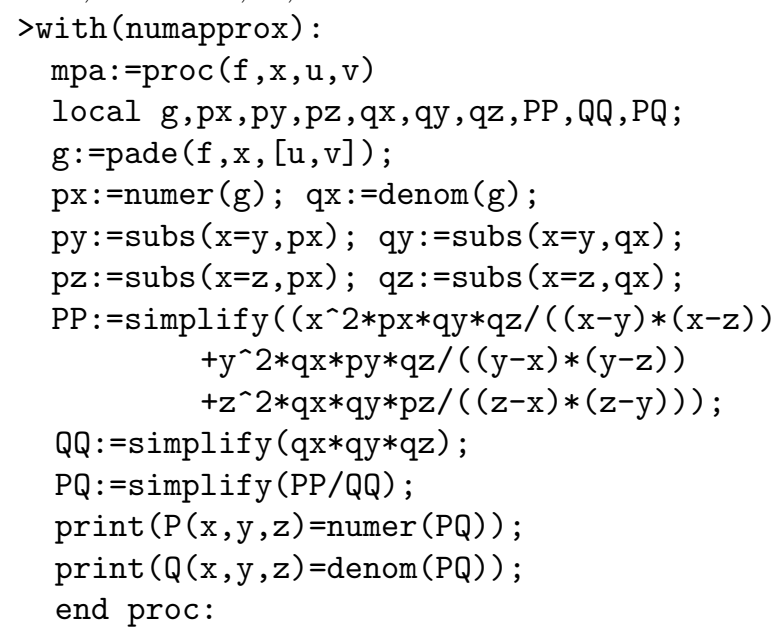


Example 4.2. A multivariate form of the logarithm series is

$$
L(\underline{x})=\sum_{j_{1}, j_{2}, \ldots, j_{m} \geq 0, j_{1}+j_{2}+\cdots+j_{m} \geq 1} \frac{x_{1}^{j_{1}} x_{2}^{j_{2}} \cdots x_{m}^{j_{m}}}{j_{1}+j_{2}+\cdots+j_{m}} .
$$

It is a pseudo-multivariate function with

$$
h(z)=\sum_{n=0}^{\infty} \frac{z^{n+1}}{n+1}=-\ln (1-z) .
$$

Running mpa(ln $(1-x), x, 2,3)$ gives

$$
\begin{aligned}
P(x, y, z)= & 729000 x+136404 x^{2} y z^{2}+729000 y+136404 x^{2} y^{2} z \\
& +136404 x y^{2} z^{2}-601128 x^{2} y z \\
& +1812780 x y z-601128 x y z^{2}+729000 z-32841 x^{2} y^{2} z^{2}-601128 x y^{2} z \\
& -461700 z^{2}-461700 y^{2}-1287900 y z+523260 y^{2} z+523260 y z^{2} \\
& -115830 y^{2} z^{2}-1287900 x y+523260 x y^{2}+523260 x^{2} y-115830 x^{2} y^{2} \\
& -461700 x^{2}-1287900 x z+523260 x z^{2}+523260 x^{2} z-115830 x^{2} z^{2},
\end{aligned}
$$

and

$Q(x, y, z)=\left(90-102 x+21 x^{2}+x^{3}\right)\left(90-102 y+21 y^{2}+y^{3}\right)\left(90-102 z+21 z^{2}+z^{3}\right)$.

Then the $(M, N)$ general order multivariate Padé approximant to the function

$$
L(x, y, z)=\sum_{j_{1}, j_{2} j_{3} \geq 0, j_{1}+j_{2}+j_{3} \geq 1} \frac{x^{j_{1}} y^{j_{2}} z^{j_{3}}}{j_{1}+j_{2}+j_{3}}
$$

on the index set $E$ is $P(x, y, z) / Q(x, y, z)$, with

$$
\begin{aligned}
N & =\left\{\left(j_{1}, j_{2}\right): 0 \leq j_{1}, j_{2}, j_{3} \leq 3\right\}, \\
M & =\left\{\left(j_{1}, j_{2}\right): 0 \leq j_{1}, j_{2}, j_{3} \leq 2, j_{1}+j_{2}+j_{3} \leq 6\right\}, \\
E & =\left\{\left(j_{1}, j_{2}\right): 0 \leq j_{1}+j_{2} \leq 5\right\} .
\end{aligned}
$$

For some multivariate series, we may not have the one variable function $h(z)$ explicitly, only by its series representation. In this case, we again use mpa ( $f$, $\mathrm{x}, \mathrm{u}, \mathrm{v})$ to compute the $(u, v)$ Padé approximant to $h(z)$ from the partial sum of degree $u+v$ and then use Theorem 2.2 to compute the $(M, N)$ general order multivariate Padé approximant to the multivariate series on the index set $E$ for any given positive integers $u, v$. We now apply this method to the Lauricella function.

Example 4.3. Our last example is the Lauricella function (see also [1, 17, 10])

$$
\begin{aligned}
F_{D}^{(m)} & \left(a, b_{1}, \ldots, b_{m} ; c ; x_{1}, \ldots, x_{m}\right) \\
& =\sum_{j_{1}, j_{2}, \ldots, j_{m}=0}^{\infty} \frac{(a)_{j_{1}+\cdots+j_{m}}\left(b_{1}\right)_{j_{1}} \cdots\left(b_{m}\right)_{j_{m}}}{(c)_{j_{1}+\cdots+j_{m}} j_{1} ! \cdots j_{m} !} x_{1}^{j_{1}} \cdots x_{m}^{j_{m}},
\end{aligned}
$$

where $(\alpha)_{n}$ is the Pochammer symbol defined by

$$
(\alpha)_{n}:=\left\{\begin{array}{cc}
\alpha(\alpha+1) \cdots(\alpha+n-1), & n \geq 1 \\
1, & n=0 .
\end{array}\right.
$$


When $b_{1}=\cdots=b_{m}=1$,

$$
F_{D}^{(m)}\left(a, 1, \ldots, 1 ; c ; x_{1}, \ldots, x_{m}\right)=\sum_{j_{1}, j_{2}, \ldots, j_{m}=0}^{\infty} \frac{(a)_{j_{1}+\cdots+j_{m}}}{(c)_{j_{1}+\cdots+j_{m}}} x_{1}^{j_{1}} \cdots x_{m}^{j_{m}},
$$

which is a pseudo-multivariate function with

$$
h(z)=\sum_{k=0}^{\infty} \frac{(a)_{k}}{(c)_{k}} z^{k} .
$$

We can use Theorem 2.2 and procedure mpa to compute the $(M, N)$ general order multivariate Padé approximant to $F_{D}^{(m)}\left(a, 1, \ldots, 1 ; c ; x_{1}, \ldots, x_{m}\right)$. For example, if $c=5, a=3$, and $m=3$, then

$$
F_{D}^{(3)}(3,1,1,1 ; 5 ; x, y, z)=\sum_{j_{1}, j_{2}, j_{3}=0}^{\infty} \frac{12}{\left(3+j_{1}+j_{2}+j_{3}\right)\left(4+j_{1}+j_{2}+j_{3}\right)} x^{j_{1}} y^{j_{2}} z^{j_{3}},
$$

which is a pseudo-multivariate function with

$$
h(z)=12 \sum_{k=0}^{\infty} \frac{1}{(3+k)(4+k)} z^{k} .
$$

Let $h_{n}(z)$ be the partial sum of degree $n$. Then $\operatorname{mpa}\left(\mathrm{h}_{5} \mathrm{z}, \mathrm{z}, 1,3\right)$ gives

$$
\begin{aligned}
P(x, y, z)= & 662872 x y^{2} z+225302 y^{2} z^{2}-278663 x^{2} y^{2} z+225302 x^{2} y^{2}+78407 x^{2} y^{2} z^{2} \\
& -278663 x y^{2} z^{2}-353780 x y^{2}-353780 y^{2} z-59930332 y+41930378 x y \\
& -353780 x^{2} y-31712555 x y z+41930378 y z+662872 x^{2} y z+662872 x y z^{2} \\
& -278663 x^{2} y z^{2}-353780 y z^{2}+225302 x^{2} z^{2}-353780 x z^{2}+94105480 \\
& -59930332 x-59930332 z+41930378 x z-353780 x^{2} z, \\
Q(x, y, z)= & 5\left(266-329 x+91 x^{2}+x^{3}\right)(266-329 y \\
& \left.+91 y^{2}+y^{3}\right)\left(266-329 z+91 z^{2}+z^{3}\right) .
\end{aligned}
$$

The $(M, N)$ general order multivariate Padé approximant to the function $F_{D}^{(3)}(3,1,1,1 ; 5 ; x, y, z)$ on the index set $E$ is $P(x, y, z) / Q(x, y, z)$ with

$$
\begin{aligned}
N & =\left\{\left(j_{1}, j_{2}, j_{3}\right): 0 \leq j_{1}, j_{2}, j_{3} \leq 3\right\}, \\
M & =\left\{\left(j_{1}, j_{2}, j_{3}\right): 0 \leq j_{1}, j_{2}, j_{3} \leq 2, j_{1}+j_{2}+j_{3} \leq 6\right\}, \\
E & =\left\{\left(j_{1}, j_{2}\right): 0 \leq j_{1}+j_{2}+j_{3} \leq 4\right\} .
\end{aligned}
$$

\section{REFERENCES}

[1] P. Appell and J. Kampé de Fériet, Fonctions hypergéométriques et hypersphériques: polynômes d'Hermite, Paris: Gauthier-Villars, 1926.

[2] G. A. Baker, Jr. and P. Graves-Morris, Padé approximants, Part I: Basic Theory, Encyclopedia of Mathematics and Its Applications 13 (Addison-Wesley, Reading, MA, 1981). MR635619 (83a:41009a)

[3] A. Cuyt, How well can the concept of Padé approximant be generalized to the multivariate case?, J. Comp. Appl. Math. 105 (1999) 25-50. MR1690577 (2000e:41029)

[4] A. Cuyt, K. Driver, and D. Lubinsky, A direct approach to convergence of multivariate nonhomogeneous Padé approximants, J. Comp. Appl. Math. 69 (1996), 353-366. MR1395293 (97g:41018)

[5] A. Cuyt, K. Driver, and D. Lubinsky, Kronecker type theorems, normality and continuity of the multivariate Padé operator, Numer. Math. 73 (1996), 311-327. MR1389490 (97g:41019) 
[6] A. Cuyt, J. Tan, and P. Zhou, General order multivariate Padé approximants for pseudomultivariate functions, Math. Comput. 75 (2006), 727-741. MR2196989 (2006i:41013)

[7] G. Lauricella, Sulla funzioni ipergeometriche a più variabili, Rend. Circ. Math. Palermo 7(1893), 111-158.

[8] M. Reimer, Constructive theory of multivariate functions, Manheim. Wien. Zürich, Wissenschaftsverlag, 1990. MR 1115901 (92m:41003)

[9] J. Stoer and R. Bulirsch, Introduction to Numerical Analysis, Springer-Verlag, New York, 1993. MR1295246(95i:65006)

[10] J. Tan and P. Zhou, On the finite sum representations of the Lauricella function $F_{D}, A d v$. Comp. Math. 23 (2005), 333-351. MR 2137460 (2006e:33020)

[11] P. Zhou, More examples on general order multivariate Padé approximants for pseudomultivariate functions, Electron. Trans. Numer. Anal. 25 (2006), 302-308. MR2280379 (2007k:41035)

Department of Mathematics, Statistics and Computer Science, St. Francis Xavier University, Antigonish, NS, Canada, B2G 2W5

E-mail address: pzhou@stfx.ca

Department of Mathematics and Computer Science, University of Antwerp, MiddelheimlaAn 1, B-2020 Antwerpen, Belgium

E-mail address: annie.cuyt@ua.ac.be

Institute of Applied Mathematics, Hefei University of Technology, 193 Tunxi Road, 230009 Hefei, People's Republic of China

E-mail address: jqtan@mail.hf.ah.cn 